\title{
Vibroacoustic Tailoring of a Rod-Stiffened Composite Fuselage Panel with Multidisciplinary Considerations
}

\author{
Albert R. Allen* and Adam Przekop $¥$ \\ NASA Langley Research Center, Hampton, Virginia 23681
}

\begin{abstract}
DOI: $10.2514 / 1 . C 033071$
An efficient multi-objective design tailoring procedure seeking to improve the vibroacoustic performance of a fuselage panel while maintaining or reducing weight is presented. The structure considered is the pultruded rod stitched efficient unitized structure, a highly integrated composite structure concept designed for a noncylindrical, next-generation flight vehicle fuselage. Modifications to a baseline design are evaluated within a six-parameter design space including spacing, flange width, and web height for both frame and stringer substructure components. The change in sound power radiation attributed to a design change is predicted using finite-element models sized and meshed for analyses in the $500 \mathrm{~Hz}, 1 \mathrm{kHz}$, and $2 \mathrm{kHz}$ octave bands. Three design studies are carried out in parallel while considering a diffuse acoustic field excitation and two types of turbulent boundary-layer excitation. Kriging surrogate models are used to reduce the computational costs of resolving the vibroacoustic and weight objective Pareto fronts. The resulting Pareto optimal designs are then evaluated under a static pressurization ultimate load to assess structural strength and stability. Results suggest that choosing alternative configurations within the considered design space can reduce weight and improve vibroacoustic performance without compromising strength and stability of the structure under the static load condition considered, but the tradeoffs are significantly influenced by the spatial characteristics of the assumed excitation field.
\end{abstract}

\section{Introduction}

$\mathbf{S}$ TUDIES presented in this paper were conducted within the framework of NASA's Environmentally Responsible Aviation (ERA) Project. ERA is exploring the feasibility, benefits, and technical risk of advanced vehicle configurations and enabling technologies that will reduce the impact of aviation on the environment. One aspect of this pursuit is the development of a lighter, more robust airframe technology that will enable unconventional aircraft configurations, such as the hybrid wing-body (HWB) [1] . A primary structural concept being pursued under ERA is the pultruded rod stitched efficient unitized structure (PRSEUS) concept (see Fig. 1). The PRSEUS construction uses a highly integrated design approach with stitched and cocured skin and substructure components to provide exceptional strength and robust damage tolerance. Manufacturing details of PRSEUS are described in detail in previous reports [2-4].

The $\overline{\mathrm{P}} \mathrm{R}$ SEUS concept has been shown to meet the static performance requirements of the HWB flat-sided pressure cabin design while also bearing significant wing loads [5]. However, due to the HWB vehicle configuration and PRSEUS properties, excessive interior noise is a concern. Therefore, there is an incentive to incorporate vibroacoustic considerations during early design stages to reduce additional mass in the form of acoustic treatments or otherwise avoid late-stage redesigns due to interior noise problems. Because the most efficient vibroacoustic tailoring can affect the primary aircraft structure, it needs to be performed under constraints dictated by the flight load requirements. Within such a framework, the vibroacoustic tailoring becomes a multi-objective task.

The goal of the present work is to develop a vibroacoustic tailoring procedure applicable to airframe structures that is appropriate for the early stages of the airframe design process. To achieve this objective,

Received 22 July 2014; revision received 2 December 2014; accepted for publication 22 December 2014; published online 13 March 2015. This material is declared a work of the U.S. Government and is not subject to copyright protection in the United States. Copies of this paper may be made for personal or internal use, on condition that the copier pay the $\$ 10.00$ per-copy fee to the Copyright Clearance Center, Inc., 222 Rosewood Drive, Danvers, MA 01923; include the code $1533-3868 / 15$ and $\$ 10.00$ in correspondence with the CCC.

*Aerospace Research Engineer, Structural Acoustics Branch; albert.r .allen@nasa.gov.

${ }^{\dagger}$ Aerospace Research Engineer, Structural Mechanics and Concepts Branch; adam.przekop@ nasa.gov. Senior Member AIAA. it is desirable that 1) the tailoring is linked to existing tools and models customary in structural design, 2) the procedure balances computational fidelity and efficiency, and 3 ) the procedure provides the ability to modify objectives without the need to repeat the entire analysis as the vehicle configuration evolves during the design process.

To fulfill the previous objectives, the following approach is undertaken. First, the results from a previous vehicle level study [2] are scrutinized to determine the primary critical flight load in the section of the airframe considered. The ability of the structure to support this load will ultimately constrain a portion of the design space considered. Next, finite-element (FE) based vibroacoustic analysis is performed using a commercial FE software routinely used for structural sizing [6]. Because high-fidelity FE models for vibroacoustic analysis can be computationally expensive, surrogate models are implemented to gain computational efficiency [7]. Generally speaking, a surrogate model is a fitted function or lookup table describing a response as a function of variational parameters. It is used in lieu of time-consuming numerical analyses or costly experiments once the initial costs of surrogate model development are addressed. To an extent, the use of surrogate models also facilitates the reassessment of the design space upon modification of the tailoring objectives without repeating the entire modeling process.

In the current design, tailoring effort both vibroacoustic performance and weight are considered as objectives. Although acoustic treatment of the primary structure is not in the scope of the current work, it can be easily envisioned that vibroacoustic performance of the primary structure and the weight of secondary acoustic treatment can also be traded to achieve the best overall vibroacoustic performance. Such an objective has the additional benefit of providing a less constrained space for other design criteria. In lieu of interior noise predictions requiring a full cabin model, a relative insertion loss (IL) metric describing the sound power radiated from one panel configuration relative to a baseline is used to judge vibroacoustic performance. Frequencies within the $500 \mathrm{~Hz}, 1 \mathrm{kHz}$, and $2 \mathrm{kHz}$ speech interference octave bands are considered in an effort to improve broadband performance. The weight metric of a panel configuration is quantified by the areal density of the panel. The static performance of vibroacoustically improved panel configurations are evaluated in subsequent analyses.

The PRSEUS panel design space considered in this study consists of six parameters including spacing, flange width, and web height for both frame and stringer substructure components. FE models of PRSEUS panels representing configuration changes with 

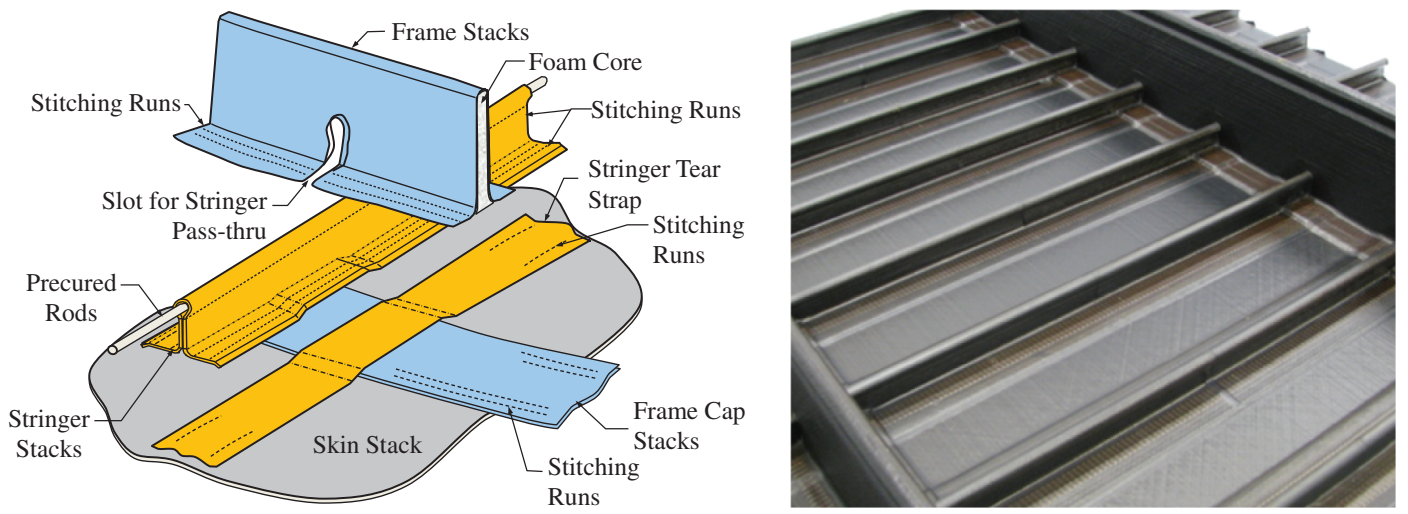

Fig. 1 PRSEUS concept exploded view (left) and image of a fabricated PRSEUS panel (right).

the required fidelity are programmatically generated. A vibroacoustic modal domain method employing a Rayleigh integral radiation impedance approximation is used to determine radiated sound power due to either turbulent boundary layer (TBL) or diffuse acoustic field (DAF) excitations. The rationale for including both types of excitation is discussed latter.

Using a deterministic FE-based analysis method to evaluate broadband vibroacoustic performance tends to be computationally expensive. A significantly less computationally expensive approach would entail use the energy diffusion method known as statistical energy analysis (SEA). However, the strong coupling between skin and substructure components in PRSEUS, which is stitched and cocured along substructure flanges, has been found to pose a difficulty for SEA in the frequency range considered [8]. Arguably, confident use of SEA in this context would require deterministic analyses to support SEA model development, especially when geometry and location of individual laminate components, such as frame and stringer flanges, are variational parameters comprising the design space. This negates much of the computational benefits of using SEA in the first place while maintaining the need for specialized knowledge in SEA modeling. Consequently, the need to maintain deterministic modeling detail while keeping the problem tractable prompted the use of a surrogate modeling technique. To accomplish this, surrogate models describing weight and IL metrics as functions of the six considered parameters were developed using a kriging method [7]. A stepped Latin sampling procedure was used to generate an amount of panel configurations to sufficiently converge the surrogate models. An evolutionary algorithm was then used to rapidly determine the Pareto optimal designs by querying the design space with the surrogate models. It is worthwhile to note that use of surrogate models has the additional benefit of providing efficient means to resolve further Pareto sets as design constraints change (typically become more confined) during the evolution of the vehicle design.

The static performance of the resulting Pareto designs is subsequently evaluated by applying a critical design ultimate load, which for the section of vehicle under consideration is determined to be a cabin pressure load. Static nonlinear FE analyses are used in the evaluations, and the resulting strains are compared against their allowables to produce margin-of-safety metrics.

Optimization of aerospace fuselage configurations for vibroacoustic performance has been of interest for at least two decades. Cunefare et al. previously developed an optimization design tool and showed an example of a two-objective weight and interior noise optimization of a metallic cylindrical section subject to tonal excitation [9]. They showed that the interior noise could be affected by simply varying skin thickness longitudinally or circumferentially and, more importantly, that the design of the primary structure can influence the fuselage vibroacoustics. Fernholz [10] and Robinson [11] addressed a 25-Hz-wide band near a peak in the interior noise level of a Beech Starship composite fuselage model due to point excitations. By optimizing laminate orientation, an inherently weight neutral approach, they were able to reduce the noise level by about
$4 \mathrm{~dB}$ but cautioned that a band-limited optimization may lead to noise amplification outside the considered band. Robinson later looked at optimizing a cylindrical fuselage composed of an aluminum honeycomb core and facesheet sandwich structure where the core thickness was varied as a cosine series in the circumferential direction and the excitation of interest was a TBL [11]. More recently, Joshi et al. addressed the TBL-excited acoustic response and weight of straight and curvilinearly stiffened metallic panels up to $1 \mathrm{kHz}$ in a multi-objective optimization while also considering static performance constraints [12].

The primary noise source considered is the fluctuating wall pressure due to a flow-induced TBL at flight condition. Miller et al. have recently given a review of TBL models relevant for fuselage vibroacoustic analysis [13]. Significant variations in the modeled fluctuating pressure field were noted, especially in the low-wavenumber region, where coupling with the fuselage dynamics is typically most active. In this paper, two TBL formulations with different low-wave-number characteristics were included to assess the impact of TBL model selection on the design tailoring outcome. Because of the difficulty of subjecting a fuselage test panel to a TBL excitation in a laboratory environment, the acoustical performances of fuselage panels are frequently experimentally evaluated in a transmission loss (TL) facility using DAF excitation. A few authors have previously shown differences in the response and radiation of fuselage panels when subject to TBL and DAF excitations. Mathur et al. showed differences in the TL of a flat unstiffened aluminum panel when subject to DAF and TBL excitations [14]. The effect of adjusting frame spacing in an aluminum-stiffened panel subject to DAF and TBL excitation was shown to diverge by Orrenius et al., suggesting that misrepresentation of the excitation field during a preliminary design stage might lead to an improved laboratory concept that actually introduces more noise in flight than the initial configuration [15]. In another study by Collery et al., the efficiency of damping treatments applied to a plate was shown to vary when subjected to DAF and TBL excitations, suggesting that laboratory-based DAF evaluations of damping treatments may lead to unnecessary weight due to overdesign [16]. Given the experimental availability and widespread use of DAF excitation, it was of interest to study a DAF excitation condition in addition to two TBL excitation conditions.

The paper begins with a description of the baseline PRSEUS panel and is followed by a description of the design space considered. The procedures used in determining vibroacoustic performance and structural viability under a limiting static pressurization load are then described. In the following section, the design tailoring procedure is discussed in three parts: 1) development of the surrogate models, 2) determination of the weight and vibroacoustic performance Pareto optimal configurations, and 3) structural viability assessments of the resulting Pareto optimal configurations. Finally, the findings are summarized in a conclusions section.

\section{PRSEUS Concept and Design Space Definition}

PRSEUS is a composite fuselage concept integrating stiched and cocured stringer and frame stiffening components. The concept takes 
advantage of an efficient load bearing substructure composed of uninterrupted pultruded rod stringers and foam-core blade frames held in place with carbon-laminate overwraps and tear straps. An exploded view of the concept is shown in Fig. 1 .

The configuration and material properties of the baseline structure considered here are based on a previously studied test panel representative of the HWB minimum-gauge pressurized cabin sidewall [8]. This structure consisted primarily of carbon-fiber warpknit fabric laminas preassembled into 1.32 -mm-thick laminates with stacking sequence and orientations (degrees) of $\left[45,-45,0_{2}, \overline{90}\right] \mathrm{S}$. The skin was composed of a single stack, whereas a mixture of single and multiple stacks were used for the frame and stringer overwraps and tearstraps. For reference, the direction of higher modulus in the single stack skin was parallel with the frames and the stringers run fore to aft for the upper cabin fuselage region considered here. Assumed material properties used for modeling purposes are shown in Table 1, and stiffener cross-section details are shown in Fig. 2.

The désign space considered was expanded into a hypervolume around the baseline configuration along six dimensions pertaining to the six substructure geometry parameters. Table $\underline{2}$ provides the variational parameters chosen and their ranges. Manufacturability and structural stability considerations were taken into account when designating the parameter ranges. Stringer spacing and flange widths were limited at the low end to avoid overlapping of neighboring stringer flanges and to ensure at least $25 \mathrm{~mm}$ width of skin region in the bays. In contrast, frame and stringer spacings were limited at the high end to avoid solver instabilities noticed during preliminary static load analyses, which typically implied poor static performance due to frame or stringer buckling. Similar precautions were used to determine the lower limits of the substructure heights.

An object-oriented framework was developed in MATLAB to quickly generate, solve, and postprocess FE-based models for particular parameter values. To reduce model complexity, details such as laminate radii at the substructure webs and the pinch detail near the frame tops were not represented. The generated model consisted primarily of composite shell elements representing the skin, flanges, and stringer and frame webs and beam elements representing the pultruded rod. Details of the model sizing, boundary conditions, and analyses performed for the performance metrics of interest are described in the following section.

\section{Performance Metrics}

\section{A. Vibroacoustic Performance}

The vibroacoustic performances of new PRSEUS configurations were evaluated by predicting the insertion loss (IL) attributed to a design change relative to the baseline configuration. Given equal fluid conditions and excitation fields for each case, this is restated as the decibel ratio of the radiated sound power $P_{\text {rad }}$ exhibited by a modified (2) configuration relative to the baseline (1) by

$$
\mathrm{IL}=10 \log _{10}\left(\frac{S_{2} P_{\mathrm{rad}, 1}}{S_{1} P_{\mathrm{rad}, 2}}\right)
$$

\section{Table 1 Material properties used for PRSEUS components}

\begin{tabular}{lcc}
\hline \hline Variable & Description & Value \\
\hline \multicolumn{3}{c}{ Single stack laminate (orthotropic) } \\
$E_{11}$ & One-direction elastic modulus & $67.2 \mathrm{GPa}$ \\
$E_{22}$ & Two-direction elastic modulus & $33.5 \mathrm{GPa}$ \\
$\nu_{12}$ & Poisson ratio & 0.40 \\
$G_{12}$ & In-plane shear modulus & $16.3 \mathrm{GPa}$ \\
$\rho$ & Density & $1578 \mathrm{~kg} / \mathrm{m}^{3}$ \\
& Pultruded stringer rod & $138.6 \mathrm{GPa}$ \\
$E$ & Elastic modulus \\
$\rho$ & Density & $1578 \mathrm{~kg} / \mathrm{m}^{3}$ \\
& Foam frame core & \\
\hline & Elastic modulus & $130 \mathrm{MPa}$ \\
$\rho$ & Density & $110 \mathrm{~kg} / \mathrm{m}^{3}$ \\
\hline \hline
\end{tabular}
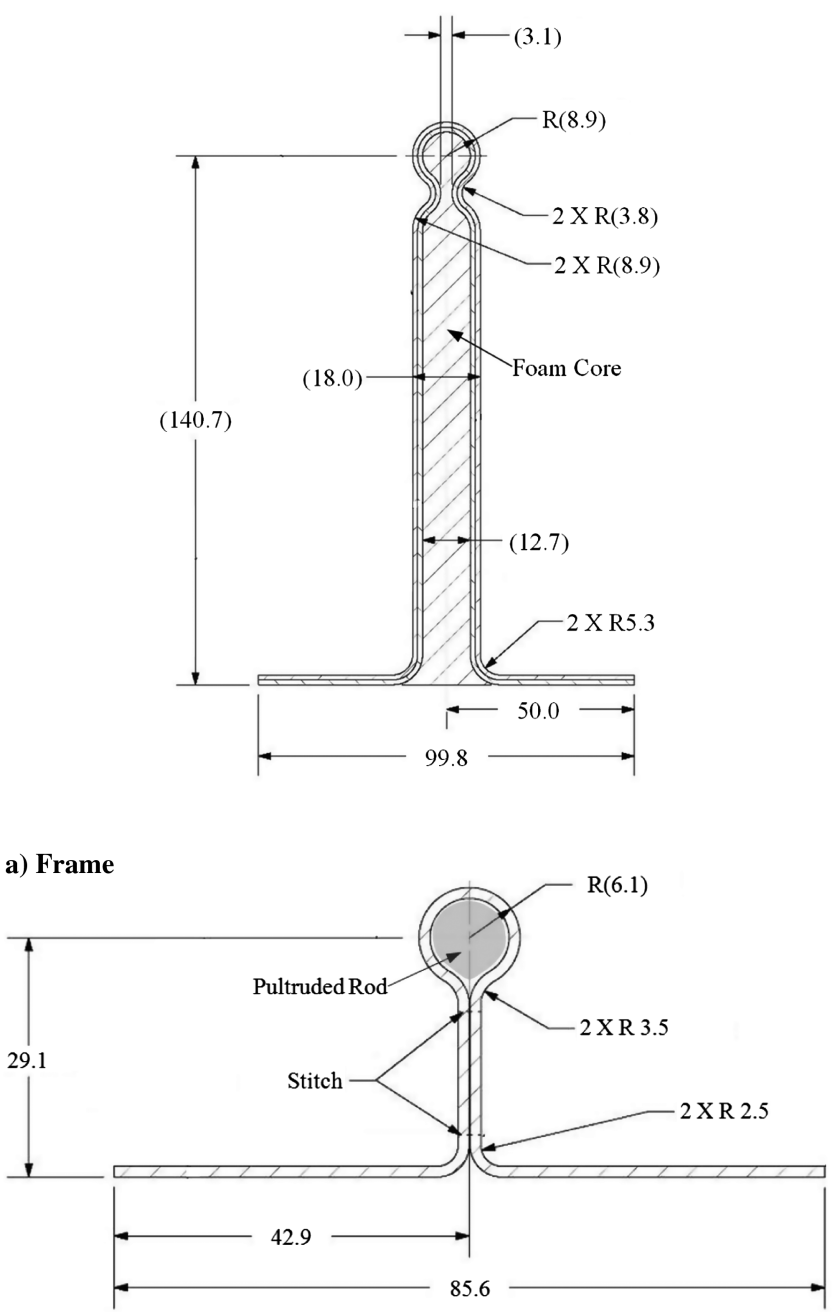

b) Stringer

Fig. 2 Baseline configuration substructure cross-section details (in millimeters). Note: cross sections not drawn to scale.

where the radiating surface areas $S$ are supplied to account for variations in panel size due to frame and stringer spacing variational parameters (refer to Table 2).

An FE modal domain method was used to estimate the sound power radiated from the interior surface of the panel due to an exterior surface excitation. The equations of motion relating forces $\{F\}$ to displacements $\{x\}$ can be written in the spatial domain as

$$
\left[-\omega^{2} \boldsymbol{M}+j \omega \boldsymbol{Z}_{f}+(1+j \eta) \boldsymbol{K}\right]\{x\}=\{F\}
$$

where $\boldsymbol{M}$ and $\boldsymbol{K}$ are the mass and stiffness matrices, and $\boldsymbol{Z}_{f}$ represents the acoustic radiation impedance. The size of the problem can be reduced by mapping the system of equations in Eq. (2) onto a reduced modal space given a set of orthogonal modes $\Phi$. The equation of motion is then restated as

$$
\left[\boldsymbol{k}_{d}+j \omega \boldsymbol{z}_{f}\right]\{\xi\}=\{\beta\}
$$

Table 2 Design space limits

\begin{tabular}{lccc}
\hline \hline Parameter & Baseline, $\mathrm{mm}$ & Range, $\mathrm{mm}$ & Range, \% \\
\hline Stringer spacing & 152.4 & {$[129.5,175.3]$} & {$[-15,15]$} \\
Frame spacing & 609.6 & {$[518.2,701.0]$} & {$[-15,15]$} \\
Stringer flange width & 85.6 & {$[52.2,102.7]$} & {$[-39,20]$} \\
Frame flange width & 99.8 & {$[79.9,119.8]$} & {$[-20,20]$} \\
Stringer rod height & 31.75 & {$[25.4,41.9]$} & {$[-20,32]$} \\
Frame web height & 152.4 & {$[121.9,182.9]$} & {$[-20,20]$} \\
\hline \hline
\end{tabular}


where $\xi=\Phi^{T}\{x\}$ and $\beta=\Phi^{\top}\{F\}$ are the modal domain displacements and forces; $z_{f}=\Phi^{T} Z_{f} \Phi$; and the modal domain dynamic stiffness matrix $\boldsymbol{k}_{d}$ is a diagonal matrix with terms

$$
k_{d_{i i}}=\left[-\omega^{2}+\omega_{i}^{2}\left(1+j \eta_{i}\right)\right] m_{i}
$$

The undamped, in vacuo eigenvalues $\omega_{i}^{2}$ and eigenvectors $\Phi_{i}$ were determined using a commercial FE solver [6]. Because the eigenvectors were mass normalized (i.e., $\Phi^{T} \boldsymbol{M} \Phi=\boldsymbol{I}$ ), the modal mass terms $m_{i}$ in Eq. (4) were set to unity. The modal damping $\eta_{i}$ is accounted for in the complex stiffness matrix during the modal domain operation. The acoustic impedance seen on either side of the panel was approximated using the Rayleigh integral formulation, which assumes an infinitely baffled planar structure radiating into a half-space and is given in terms of force/velocity in the spatial domain as

$$
\boldsymbol{Z}_{f}=\frac{j \omega \rho_{0} \boldsymbol{A}^{2}}{2 \pi \boldsymbol{R}} e^{(-j k \boldsymbol{R})}
$$

where $\boldsymbol{A}$ is a diagonal matrix of nodal areas, and $\boldsymbol{R}$ is the nodal separation matrix [17]. The diagonal of $\boldsymbol{Z}_{f}$ is undefined as the nodal separation goes to zero, and so these terms were replaced by the radiation impedance of a baffled piston expressed as

$$
Z=\rho_{0} c_{0} \pi \hat{r}^{2}\left((k \hat{r})^{2} / 2+j 8 k \hat{r} /(3 \pi)\right)
$$

where $\hat{r}$ is the piston radius chosen to conserve nodal area [18]. The modal domain mobility was then determined by inverting the

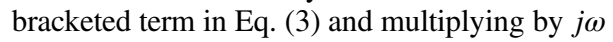

$$
\boldsymbol{y}=j \omega\left(\boldsymbol{k}_{d}+j \omega \boldsymbol{z}_{f}\right)^{-1}
$$

Interior and exterior fluid properties are accounted for in $z_{f}$; the structural characteristics are represented in the undamped eigenvectors and eigenvalues; and a frequency-independent loss factor of $2 \%$ $\left(\eta_{i}=0.02\right)$ was assumed. The modal domain velocity cross-power spectral density (CPSD) matrix is then calculated:

$$
\boldsymbol{G}_{v v}=\boldsymbol{y} \Phi^{T} \boldsymbol{G}_{F F} \Phi y
$$

for a given spatial domain force CPSD matrix $\boldsymbol{G}_{F F}$. The radiated power was then calculated from the real part of the product of force and velocity summed over all modes:

$$
P_{\mathrm{rad}}=\sum_{i=1}^{N} \sum_{j=1}^{N} \operatorname{Re}\left\{z_{f_{i j}} G_{v_{i} v_{j}}\right\}
$$

Three conditions were considered: 1) an unpressurized laboratory condition with diffuse acoustic field (DAF) excitation, 2) a pressurized cabin at cruise condition with Corcos TBL excitation, and 3) a pressurized cruise condition with a modified Corcos TBL excitation. The laboratory condition case simulated a typical TL test setup involving dual reverberation or reverberation/anechoic chambers, whereby a DAF force CPSD is applied to the exterior side nodes and is given as

$$
\boldsymbol{G}_{F F}=2\left\langle G_{p p}\right\rangle A^{2} \operatorname{sinc}(k \boldsymbol{R})
$$

where $\left\langle G_{p p}\right\rangle$ is the average room pressure PSD, which was set to $1 \mathrm{~Pa}^{2} / \mathrm{Hz}$ [19]. The factor of 2 in Eq. (10) accounts for a doubling of the mean square pressure seen at the wall due to the Waterhouse effect [20], although this drops out when calculating IL.

For the cruise condition cases, a representative region of the fuselage near the top center $20 \mathrm{~m}$ from the nose was subjected to a simulated cruise environment. A static differential pressurization of $1 P=63 \mathrm{kPa}$ was applied to the interior surface during a static nonlinear solution before the modal analysis. The pressurization step was included primarily to account for the bay stiffening effects expected in the pressurized cabin sidewall at cruise. The noise source considered at cruise was the exterior surface fluctuating pressure field due to a TBL. To model the spatial characteristic of the excitation field, the separable Corcos and modified Corcos CPSD formulations were applied and are written respectively as

$$
\Gamma\left(\xi_{1}, \xi_{2}, \omega\right)=\phi(\omega) A^{2} e^{-\frac{\left|\xi_{1}\right|}{L_{1}}} e^{-\frac{\left|\xi_{2}\right|}{L_{2}}} e^{-\frac{j \omega \xi_{1}}{U_{c}}}
$$

and

$$
\Gamma\left(\xi_{1}, \xi_{2}, \omega\right)=\phi(\omega) A^{2}\left(1+\left|\xi_{1} / L_{1}\right|\right) e^{-\frac{\left|\xi_{1}\right|}{L_{1}}} e^{-\frac{\left|\xi_{2}\right|}{L_{2}}} e^{-\frac{j \omega \xi_{1}}{U_{c}}}
$$

where $\xi_{1}$ and $\xi_{2}$ are, respectively, the spatial separations in the streamwise and cross-stream directions $[21,22]$. The low streamwise wave-number excitations exhibited by Eqs. (11) and (12) vary considerably and were thought to be representative of the variations seen in TBL CPSD formulations found in the literature [13]. It was of interest to consider the extent that differences in the low-wavenumber excitation might influence the outcome in this and similar multi-objective design studies.

The streamwise and cross-stream coherence length terms were estimated using Efimtsov's model and are given respectively as

$$
L_{1}=\delta\left[\left(\frac{a_{1} S h}{U_{c} / U_{\tau}}\right)^{2}+\frac{a_{2}^{2}}{S h^{2}+\left(a_{2} / a_{3}\right)^{2}}\right]^{-\frac{1}{2}}
$$

and

$$
L_{2}=\left\{\begin{array}{cc}
\delta\left[\left(\frac{a_{4} S h}{U_{c} / U_{\tau}}\right)^{2}+\frac{a_{5}^{2}}{S h^{2}+\left(a_{5} / a_{6}\right)^{2}}\right]^{-\frac{1}{2}} & \text { for } M<0.75 \\
\delta\left[\left(\frac{a_{4} S h}{U_{c} / U_{\tau}}\right)^{2}+a_{7}^{2}\right]^{-\frac{1}{2}} & \text { for } M>0.9
\end{array}\right.
$$

where $a_{1}$ to $a_{7}$ are, respectively, $0.1,72.8,1.54,0.77,548,13.5$, and 5.66 [23]. The frequency dependence of Eqs. (13) and (14) are attributed to the Strouhal number $S h=\omega \delta / U_{\tau}$; the TBL convective velocity was assumed to be $70 \%$ of the freestream velocity, written as $U_{c}=0.7 U_{\infty}$; and the flight condition was specified with a freestream velocity of Mach 0.85, and so the expression in Eq. (14) was interpolated accordingly. An approximation was made by assuming a white-in-band single-point wall pressure PSD (i.e., $\phi(\omega)=1 \mathrm{~Pa}^{2} / \mathrm{Hz}$ ). This was thought to be a benign assumption given the performance metric chosen (IL). The friction velocity $U_{\tau}$ was used while assuming a flat-plate TBL with a zero pressure gradient given by Schlichting [24] as

$$
U_{\tau}=\sqrt{\tau_{w} / \rho_{f}}
$$

where the wall shear stress term is given as

$$
\tau_{w}=0.0225 \rho_{f} U_{\infty}^{2} R e_{\delta}^{-0.25}
$$

and where the boundary-layer thickness scaled Reynolds number is expressed as

$$
R e_{\delta}=U_{\infty} \delta / \nu
$$

The kinematic viscosity $\nu$, density $\rho_{f}$, and speed of sound $c$ (used to calculate $U_{\infty}$ for the given Mach number) were determined by using standard atmosphere tables. Boundary-layer thickness and displacement thickness estimates were obtained from previous computationalfluid-dynamics analyses of the HWB-450-1L planform [25]. A summary of TBL parameters for the flight condition considered are listed in Table 3.

Experimental DAF TL measurements taken with a $1.22 \times 1.22 \mathrm{~m}$ flat PRSEUS test article at the NASA Langley Structural and Acoustic Loads Testing Facility, and predictions made using the modal domain method described here are compared in Fig. $\underline{3}$ within the considered frequency range []. Agreement with experiment was 
Table 3 Turbulent boundary-layer parameters

\begin{tabular}{lc}
\hline \hline Parameter & Value \\
\hline Mach number $M$ & 0.85 \\
Altitude $h_{\text {alt }}, \mathrm{m}$ & 10,668 \\
Speed of sound $c_{f}, \mathrm{~m} / \mathrm{s}$ & 296.6 \\
Freestream velocity $U_{\infty}, \mathrm{m} / \mathrm{s}$ & 252.1 \\
TBL convective velocity $U_{c}, \mathrm{~m} / \mathrm{s}$ & 176.5 \\
Fluid density $\rho_{f}, \mathrm{~kg} / \mathrm{m}^{2}$ & 0.381 \\
Kinematic viscosity $\nu, \mathrm{m}^{2} / \mathrm{s}$ & $3.7694 \times 10^{-5}$ \\
Temperature $T, \mathrm{~K}$ & 218.92 \\
Wall shear stress $\tau_{w}, \mathrm{~kg} / \mathrm{m} / \mathrm{s}^{2}$ & 15.23 \\
Friction velocity $U_{\tau}, \mathrm{m} / \mathrm{s}$ & 6.33 \\
TBL thickness $\delta, \mathrm{m}$ & 0.244 \\
TBL displacement thickness $\delta^{*}, \mathrm{~m}$ & 0.021 \\
\hline \hline
\end{tabular}

deemed adequate for the design tailoring effort described here. The IL spectra attributed to a design modification were also predicted while considering various excitation fields including the three fields considered here as well as a turbulent boundary layer based on a nonseperable elliptical formulation. The same general approach was taken here, although in this case, the analysis was partitioned by octave band to reduce computational expense by making use of smaller panel sizes at higher frequencies, where the effect of boundary conditions and sizing on the IL were relatively low. A breakdown of the model sizing per band is shown in Fig. 4 for the baseline configuration. Note that the panel sizing was dictated by frame and stringer count, and the overall panel dimensions varied somewhat due to frame and stringer spacing variational parameters. The effect of reduced model sizing for the 1 and $2 \mathrm{kHz}$ octave band analyses relative to the full two-frame-by-eight-stringer sizing was evaluated with Eq. (1) for the baseline configuration while considering all three conditions. The differences were found to be less than 0.3 and $0.9 \mathrm{~dB}$ for the 1 and $2 \mathrm{kHz}$ model sizings, respectively. Clamped boundary conditions were assumed along the perimeter of the in-plane skin elements but were not extended to the out-of-plane portions of the stringer and frame cross sections.

An effort was made to converge the mesh resolution to an acceptable level while also avoiding excessive runtimes and memory limitations. This was done by varying the maximum allowed element edge lengths in the baseline models and comparing $P_{\text {rad }}$ for a given mesh resolution and condition with the same results obtained from the highest mesh resolutions feasibly considered given the available computational resources, denoted as $P_{\text {rad }}^{\dagger}$ in Fig. 5. The maximum allowed element edge lengths were set to $25.4,19.1$, and $12.7 \mathrm{~mm}$ for the $500 \mathrm{~Hz}, 1 \mathrm{kHz}$, and $2 \mathrm{kHz}$ octave bands, respectively. These element sizings were thought to be an acceptable trade between

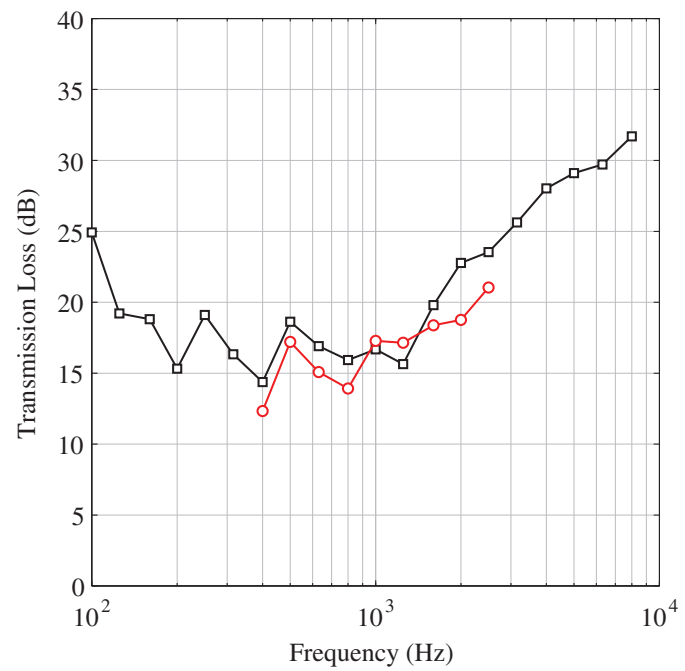

Fig. 3 Experimental DAF TL ( $\square$ ) compared with vibroacoustic FE predictions (O). accuracy and computational expense as use of smaller elements incurred a significant increase in computational expense with relatively small changes in radiated sound power. Panel modes with natural frequencies well outside the frequency ranges of each octave band were also removed from $\Phi$ before analysis to reduce unnecessary computational expense. Only modes with natural frequencies below twice the highest frequency in band were included for the $500 \mathrm{~Hz}$ octave analysis, $20 \%$ above and below the band for the $1 \mathrm{kHz}$ octave analysis, and $10 \%$ above and below the band for the $2 \mathrm{kHz}$ octave analysis. The selected frequency ranges of mode inclusion were deemed adequate when no significant changes were found (less than $0.4 \mathrm{~dB}$ ) upon comparing baseline configuration radiated sound power results with $\Phi$ truncated to those with $\Phi$ containing all modes below $4 \mathrm{kHz}$. Finally, the results were reduced to a single IL metric by simply taking the arithmetic average of the three octave IL values from Eq. (1).

\section{B. Static Strength and Stability}

A previous analysis [2] of the notional civil transport aircraft designated as BWB-5200G was used to define representative static loads acting on the panel. The ultimate cabin pressurization load of

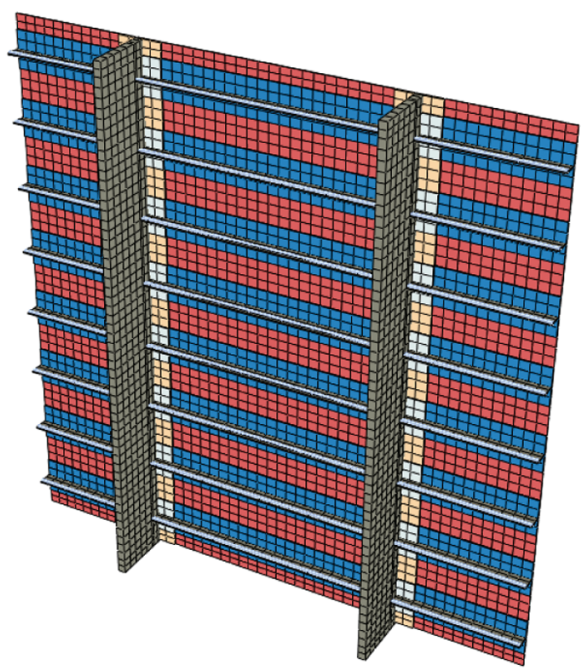

a) $500 \mathrm{~Hz}$ ( 2 frames by 8 stringers)

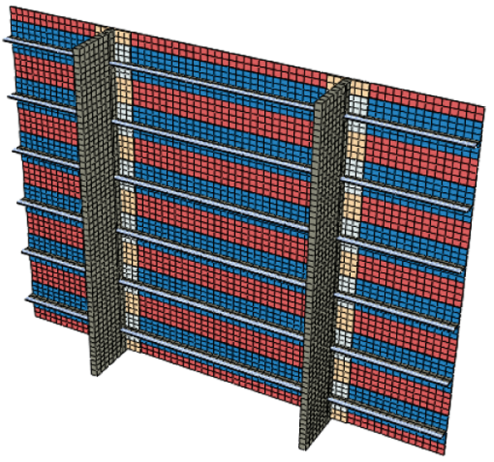

b) $1 \mathrm{kHz}$ ( 2 frames by 6 stringers)

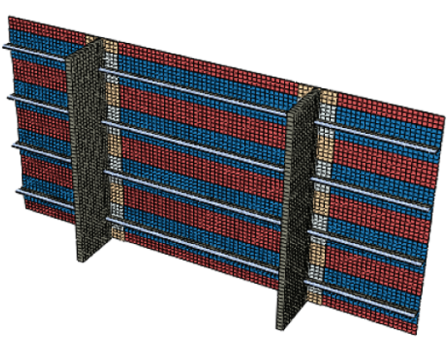

c) $2 \mathrm{kHz}$ ( 2 frames by 4 stringers)

Fig. 4 Panel sizes used for the three octave bands considered. 

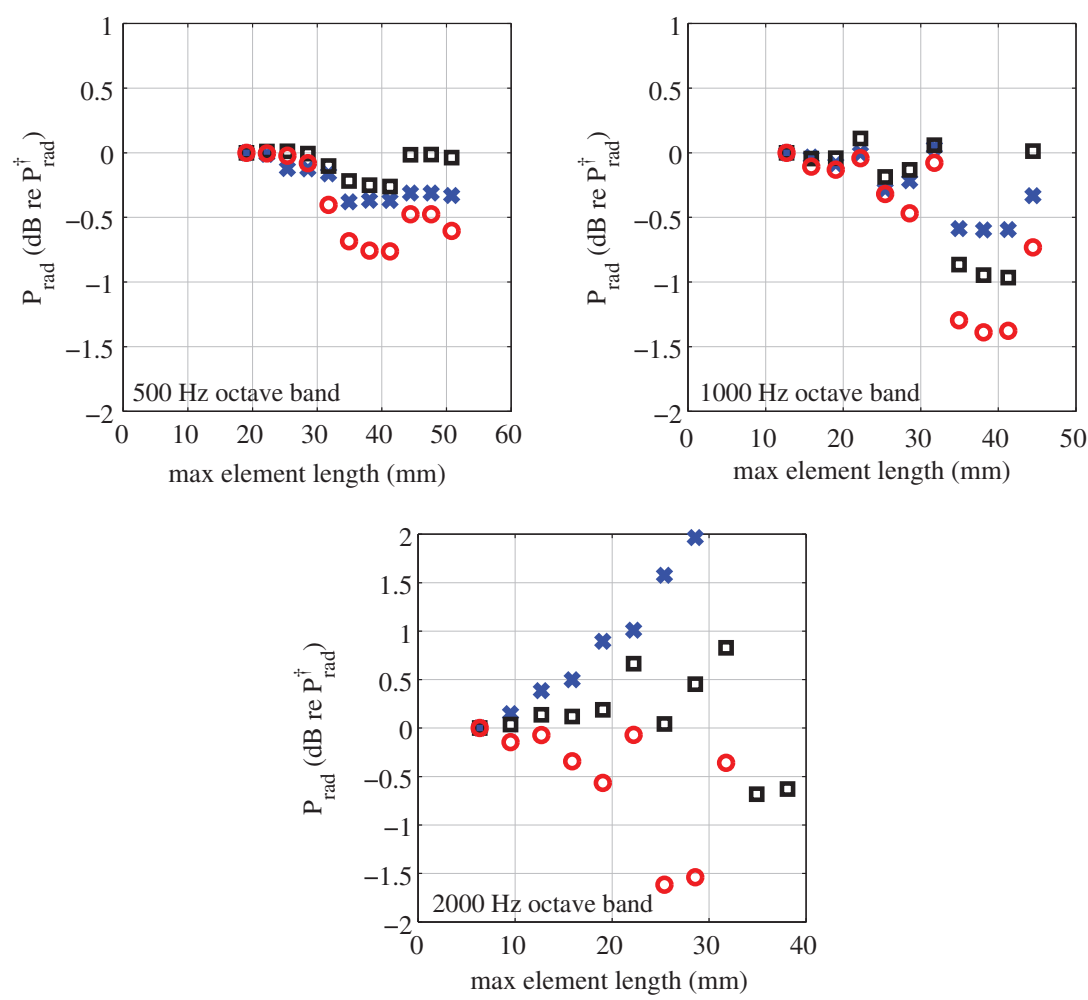

Fig. 5 Convergence of radiated sound power estimates with respect to maximum element edge length allowed in the panel FE model. Sound power radiated $\boldsymbol{P}_{\text {rad }}$ is given in decibels relative to $P_{\text {rad }}^{\dagger}$, the estimate obtained from the highest-resolution model considered for each octave band analysis. Results are shown for laboratory condition DAF (*), cruise condition Corcos (O), and modified Corcos ( $\square$ ) TBL excitations.

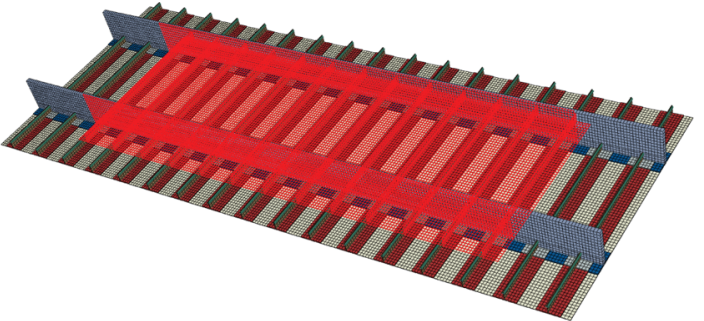

Fig. 6 Finite-element model of the pressure panel with substructure facing upward. Regions included in strain evaluation are highlighted in red.

$2 P=127 \mathrm{kPa}$ (twice the expected cabin pressurization at cruise) was found to be limiting for a majority of the fuselage panel configurations in the region of interest. Therefore, only the $2 P$ load was considered in this study. The panel size during the static analyses was chosen to mimic a 2.743 by $1.219 \mathrm{~m}$ planar pressure panel previously tested [26]. Test results and FE model predictions reported in [26] were also used to develop simplified boundary conditions that mimicked the experimental pressure vessel and clamping frame support structure. An example model is shown in Fig. $\underline{6}$, where the region of strain evaluation away from the boundaries is highlighted. Because actual boundary conditions of the PRSEUS panel would likely vary in future applications, the results obtained in the immediate vicinity of the panel boundaries were of limited interest and were excluded from the strain assessment. It was assumed that the exact boundary conditions for multibay structures are not presently known, but the panel joints can be designed such that the load introduction does not result in excessive localized strain levels.

Because the pressure was applied to the stiffened side of the panel corresponding to the aircraft interior, the panel out-of-plane deformation was toward the smooth side of the panel. Consequently, the surface (planar) portion of the panel became mostly stretched under the load, and the most inward portions of the substructure became compressed. This implied two main possible failure mechanisms: fracture in tension and loss of stability in compression.
A commercially available nonlinear solver was used to capture possible stability loss in the form of substructure buckling due to compression [].

Once the strain fields in the panel subjected to the pressurization load were computed, extreme values in tension and compression were identified and compared with the material allowables representative of the material systems used in the study, as presented in Table 4. Because the material allowables differ substantially depending on the section of the panel, a unified comparison was made by computing the margin of safety (MOS) values defined as

$$
\operatorname{MOS}_{(\min , \max )}=\frac{\epsilon_{\mathrm{allow}(\min , \max )}}{\epsilon_{(\min , \max )}}-1
$$

The MOS is equal to or greater than zero for components that maintain structural integrity under the prescribed load, and the larger the MOS value is, the more conservative the design is. Conversely, when the MOS is negative, the structural component is predicted to fail under the prescribed load.

\section{Design Tailoring Procedure}

\section{A. Development of Surrogate Model}

Kriging surrogate models were developed using the MATLAB DACE kriging toolbox to quickly resolve the vibroacoustic performance metric at any location within the design space [7]. A Latin sampling method was used to populate the surrogate model with known response values to ensure a well-distributed set of samples throughout the design space. The sampling was performed in steps to

Table 4 Material strain allowables $(\mu \epsilon)$

\begin{tabular}{lrc}
\hline \hline Region & Tension & Compression \\
\hline In-plane components & 5,900 & 4,800 \\
Substructure & 7,000 & 5,800 \\
Pultruded rod & 17,000 & 10,000 \\
\hline \hline
\end{tabular}


avoid unnecessary design evaluations once the surrogate models were adequately converged. The stepping procedure used was similar to the method described by Sallaberry et al. [27], where the sample locations from a given step are chosen away from previous sample locations to maintain the good spatial distribution of a Latin sampling, and the total number of samples $m$ is doubled each step. Model accuracy was evaluated by directly calculating the root mean square error (RMSE) attributed to response prediction $\hat{y}$ relative to known response $y$ with

$$
\mathrm{RMSE}=\sqrt{\frac{1}{m} \sum_{i=1}^{m}\left(\hat{y}\left(\boldsymbol{x}_{i}\right)-y\left(\boldsymbol{x}_{i}\right)\right)^{2}}
$$

and by predicting the RMSE as a function of the fitted surrogate model parameters evaluated within the DACE kriging toolbox. At each step, newly evaluated responses were compared with the values predicted from the surrogate model fitted using response points from all previous steps. Results of the convergence study for the surrogate models used are shown in Fig. 7. The IL metric surrogate models were converged to approximately 0.1 RMSE in terms of decibels, whereas the weight metric was converged to approximately $1 \times 10^{-4}$ RMSE in terms of area density percent change. Because the direct RMSE estimate from Eq. (19) required both new sample locations and their responses, it was not evaluated during the final step due to the additional computational expense required. Because the computational expense of calculating area density was very low, no effort was made to minimize the number of steps required to converge the weight metric surrogate model.

The predicted RMSE was found to be generally in good agreement with the direct RMSE as the sample size increased. The relatively larger error seen in the IL metric surrogate model for the modified Corcos excitation case motivated the addition of samples from one additional step relative to the other cases. This was not surprising when considering that the IL metric is influenced by structural
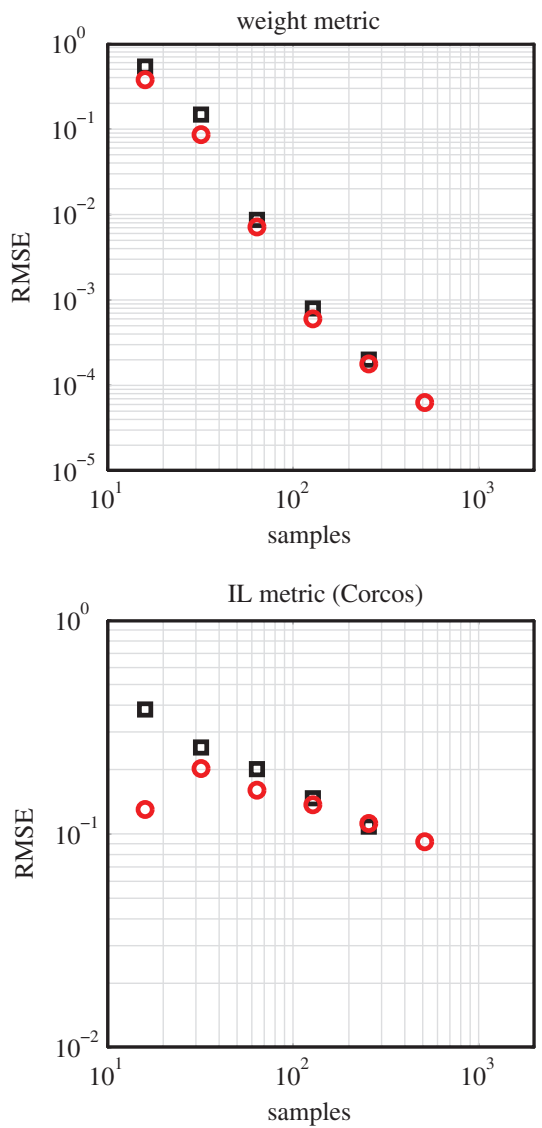

sensitivity variations in the subconvective streamwise wave numbers and that the modified Corcos model exhibits a relatively steeper slope in the same wave-number region producing larger variations in the response surface []].

\section{B. Two-Objective Optimization}

The weight and vibroacoustic objectives were then restated as a two-objective minimization problem by considering the weight metric as is and the negative of the IL metric. It was of interest to determine an array of Pareto optimal configurations for later use in design trade studies. The two objective Pareto optimal set can be described as the set of best compromises between both objectives within the design space (i.e., no improvements can be made to the Pareto optimal set within the design space without sacrificing performance in one or more objectives). The hybrid optimization algorithm known as GODLIKE (from "Global Optimum Determination by Linking and Interchanging Kindred Evaluators") was used to resolve the Pareto set by using mixed fitness algorithms in an Non-dominated Sorting Genetic Algorithm framework [28]. In GODLIKE, offspring are randomnly interchanged among different fitness algorithms at each generation to improve robustness by avoiding possible local optima convergence of any one algorithm. In this study, the genetic algorithm, differential evolution, and simulated annealing modules were used. The process was seeded with a population size of 2000 and allowed to run until convergence, which typically took on the order of 500,000 function evaluations. The computational expense of querying the surrogate models was low, and the total run times with the chosen settings were typically less than $5 \mathrm{~min}$.

The resulting Pareto optimal sets are shown for the three excitations considered in Fig. 8 . The curves generated from the Pareto sets extend into regions of improved performance relative to the baseline (lower values denote improvement) and suggest vibroacoustic improvements with no weight penalty at the $0 \%$ weight objective intersections and
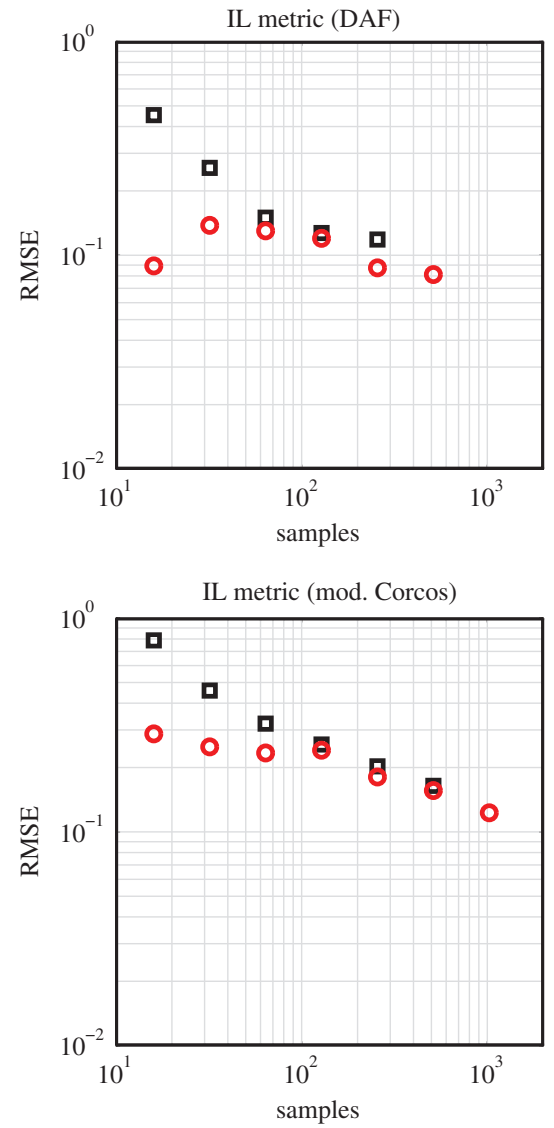

Fig. 7 Expected error (RMSE) attributed to weight and IL metric kriging surrogate model predictions vs sample size using direct ( $\square$ ) and predicted ( $($ ) estimates. 


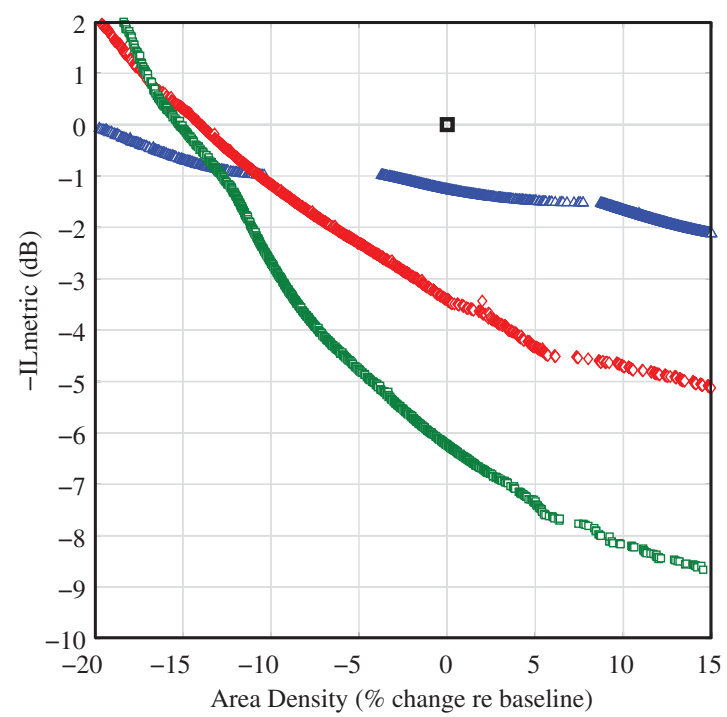

Fig. 8 Two objective Pareto optimal sets evaluated while considering laboratory condition DAF $(\triangle)$ and cruise condition Corcos $(\diamond)$ and modified Corcos $(0,0.5,0 \square)$ TBL excitations. The baseline configuration performance $(\square)$ is shown at $(0,0)$ for reference.

weight savings with no vibroacoustic penalty at the $0 \mathrm{~dB}$ IL objective intersections. It is also apparent that the prescribed design space offers varying degrees of performance gains depending on the excitation considered. The laboratory condition DAF excitation case shows relatively small performance gains (approximately $1 \mathrm{~dB}$ in terms of the IL metric) near $0 \%$ area density change, whereas the weight reduction potential at equal vibroacoustic performance extends down to $20 \%$ reduction in area density. The two cruise condition TBL excitation cases show similar Pareto front shapes, although the scale of vibroacoustic performance gains are relatively larger for the modified Corcos case over much of the front. This is attributable to the aforementioned differences in the excitation field wave-number content and was also noticed during a previous two-point study [8]. That the two TBL fronts converge near the $0 \mathrm{~dB}$ IL metric line is also supported by this explanation as the relative effect of the slope of the excitation field subconvective wave-number region would naturally diminish for configurations exhibiting little or no difference in vibroacoustic performance.

If one simply accounts for mass law effects, $a \pm 15 \%$ change in area density corresponds to an IL of approximately $\pm 1.3 \mathrm{~dB}$. However, this is hardly a reliable predictor for such a built-up panel, especially when considering that the prescribed design space allows for redistribution of mass and stiffness among components of varying vibroacoustic sensitivity, namely the in-plane flange components relative to, say, the out-of-plane frame components.

Pareto configurations located at the $0 \%$ area density change line were analyzed to provide example narrowband and $1 / 3$ octave IL results as shown in Fig. 9 . Table $\underline{5}$ provides the design parameters pertaining to Fig. 9 as well as the baseline parameters for reference. For these singular runs, the large two-frame-by-eight-stringer panel sizing was used, a maximum element edge length of $12.7 \mathrm{~mm}$ was prescribed, and all modes were included up to $4 \mathrm{kHz}$ in an attempt to provide best-fidelity final evaluations. When regarding the single value IL metric, Pareto front results from Fig. $\underline{8}$ are in quite good agreement with the $0 \%$ mass gain example results. This suggests that small errors attributed to the use of surrogate models, reduced model sizing, and reduced modal inclusion were not significant. After viewing the narrowband and $1 / 3$ octave IL results, it becomes apparent that mass neutral vibroacoustic improvements to the structure within the considered design space will be difficult to achieve for DAF situations. However, this does not preclude the notion that weight savings may be achieved without impacting vibroacoustic performance. For the cruise conditions, the narrowband and $1 / 3$ octave
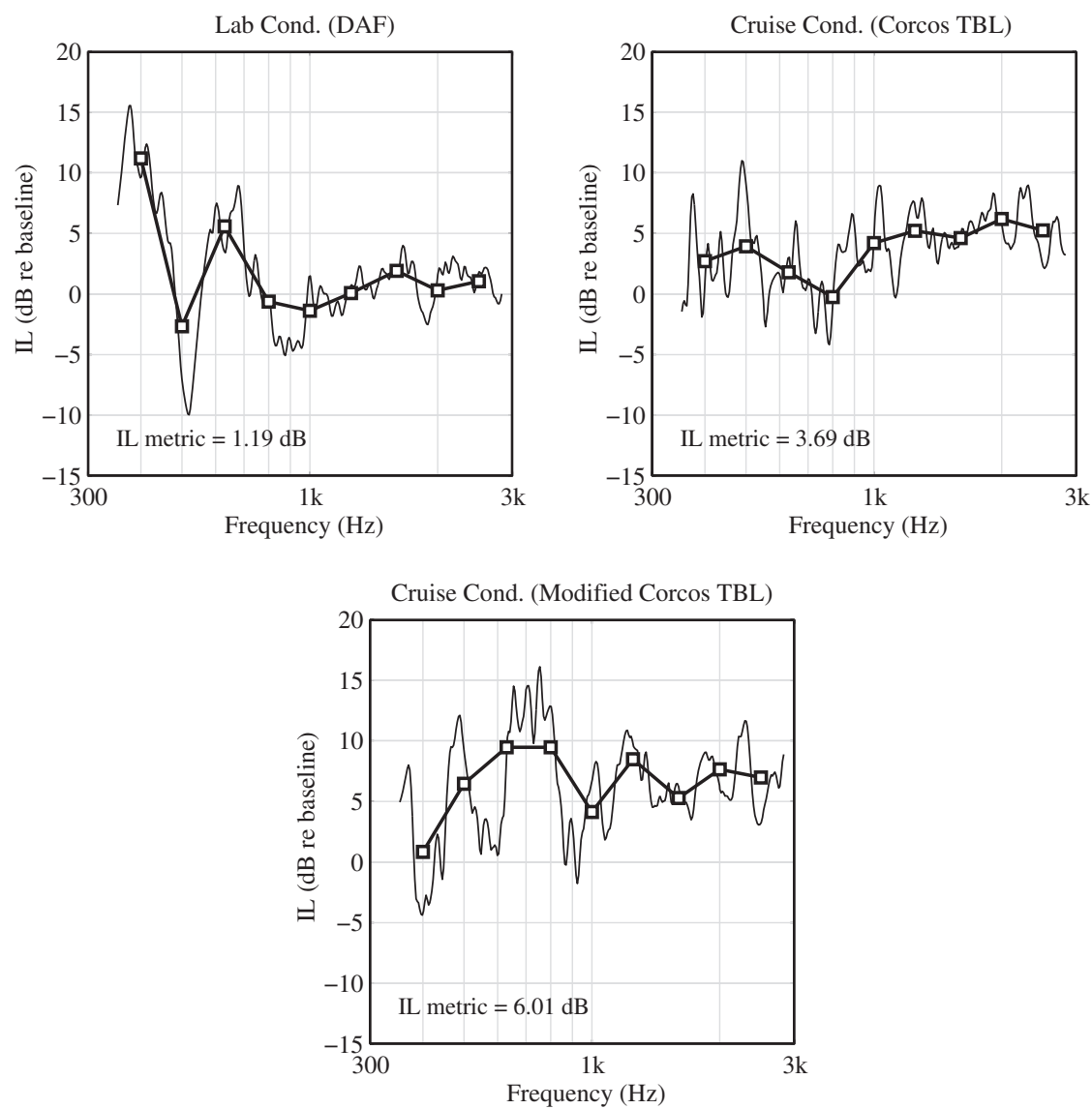

Fig. 9 Narrowband (-) and 1/3 octave ( $\square$ ) IL results for configurations located on the Pareto front at $0 \%$ area density change for the three conditions considered. The single value IL metric results corresponding to the objectives in Fig. 8 are also given. 
Table 5 Parameter values (in millimeters) for baseline and weight neutral optimum designs corresponding to Fig. 9

\begin{tabular}{|c|c|c|c|c|}
\hline$\underline{\text { Parameter }}$ & Baseline & $\begin{array}{c}\text { Lab } \\
\text { condition } \\
\text { (DAF) } \\
\end{array}$ & $\begin{array}{c}\text { Cruise } \\
\text { condition } \\
(\text { Corcos TBL }) \\
\end{array}$ & $\begin{array}{c}\text { Cruise } \\
\text { condition (modified } \\
\text { Corcos TBL) } \\
\end{array}$ \\
\hline Stringer spacing & 152.4 & 132.6 & 129.9 & 129.5 \\
\hline Frame spacing & 609.6 & 553.5 & 700.4 & 700.4 \\
\hline Stringer flange width & 85.6 & 52.4 & 102.0 & 86.9 \\
\hline Frame flange width & 99.8 & 118.9 & 80.3 & 80.6 \\
\hline Stringer rod height & 31.75 & 41.3 & 25.7 & 40.4 \\
\hline Frame web height & 152.4 & 127.4 & 122.1 & 122.1 \\
\hline
\end{tabular}

results show improvement on a largely broadband basis, where (barring a few narrowband excursions into negative values) the majority of the IL spectrum is positive.

The arrays of Pareto optimal configurations are shown in Fig. 10. The plots show the Pareto configurations as viewed along the weight objective line in terms of variational parameter percent change relative to baseline. Configurations exhibiting more than a $5 \%$ increase in area density are not of particular interest and are not shown. Upon initial inspection, dissimilar trends in the optimal configurations resulting from laboratory condition DAF and cruise condition TBL cases are noticed. For many of the parameters, especially the frame and stringer flange widths near the $0 \%$ mass change configurations, the trend is even reversed. On the contrary, the Pareto configurations resulting from the two cruise condition TBL formulations are generally similar, although there are differences in the stringer height and flange width parameters. For the majority of the parameters, especially when considering the results from the cruise condition cases, the optimization has moved the configurations toward the limits of the design space. Although physical limitations and manufacturability were considered when designating the design space limits, there may be opportunity in the future to further expand the design space. However, without considering the structure's static performance, further optimization over an expanded design space may simply move the design further into regions of infeasibility.

\section{Structural Viability Check}

Structural MOS trends of the Pareto configurations shown in Fig. 10 were subsequently investigated using the previously described model generation and analysis framework, wherein flat-panel FE models including 16 stringers and two frames were subjected to $2 P$ pressurization in nonlinear static analyses. The worst-case minimum MOS in tension and compression within the major panel components are shown in Fig. 11 for Pareto configurations resulting from the three conditions considered. Comparisons with the baseline configuration are shown as horizontal lines for reference.

When viewing the in-plane results, a predictable trend is noticed for all three conditions. Reduced weight generally corresponds with less conservative configurations with respect to both tension and compression allowables. The Pareto configurations are comparable with the baseline near $0 \%$ area density change and approach a lower MOS near 0.5 for the lowest weight configurations. For the stringer web regions, compression is found to be a limiting condition, and the Pareto configurations offer some relief for these load paths relative to the baseline. This is not surprising, given the trend toward reduced stringer spacing for the three conditions. The height of the stringer is also somewhat significant here when noticing that the increased compression (reduced MOS) correlates with increased stringer height for the cruise condition designs in particular. Only the compression results were shown for the rod regions because the tension MOSs were quite large and, consequently, not of considerable interest. The trend toward increased conservativeness is seen here and relates closely with the stringer web compression results in both character and explanation.
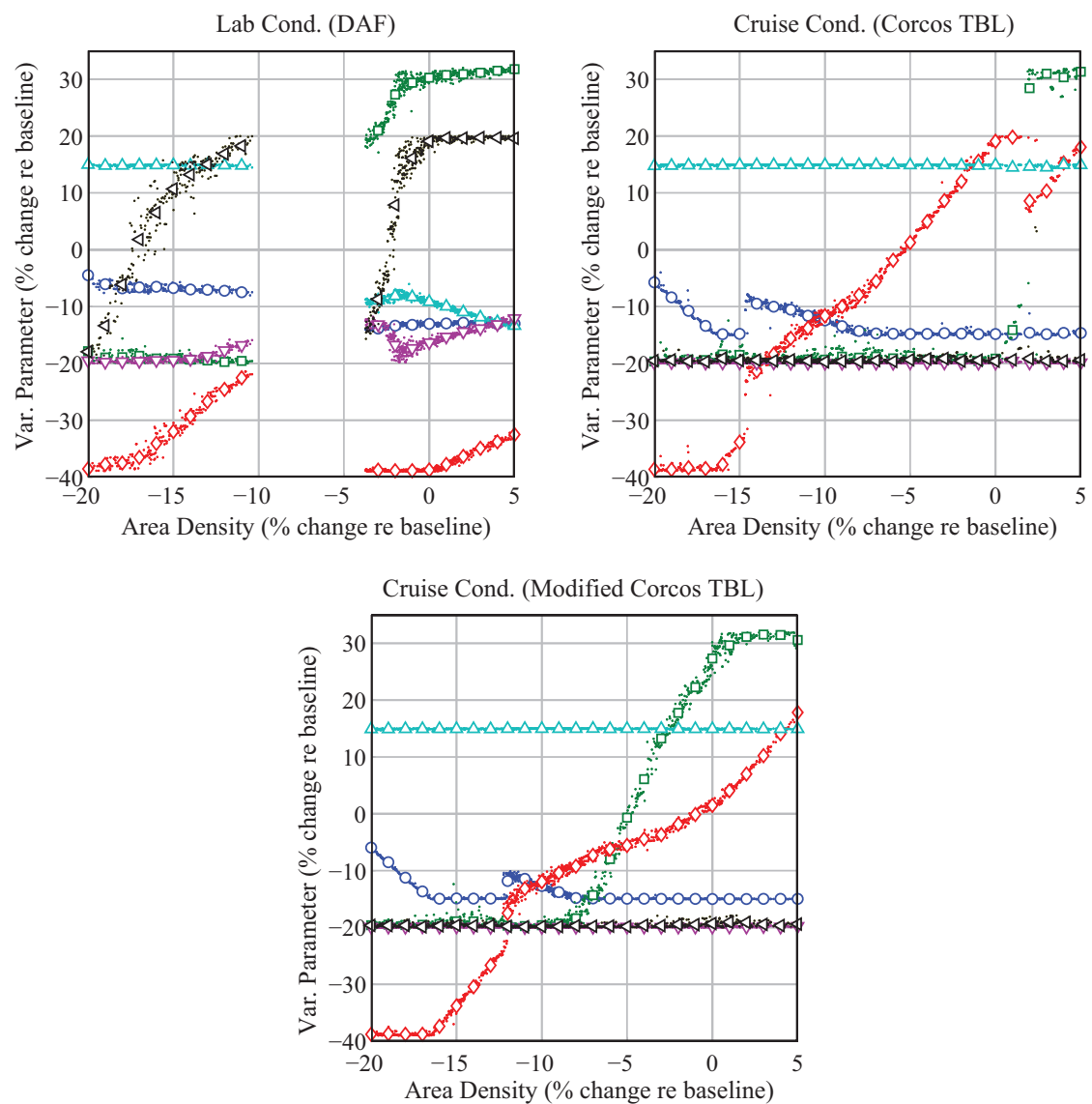

Fig. 10 Variational parameters of the Pareto optimal configurations from Fig. 8 plotted along a portion of the weight objective line for the three conditions considered. Averaged values of stringer spacing $(\circ)$, stringer height $(0,0.5,0 \square)$, stringer flange width $(\diamond)$, frame spacing $(\triangle)$, frame height $(\nabla)$, and frame flange width $(\triangleleft)$ parameters in percent change relative to baseline are plotted with dots showing the spread of Pareto data points. 

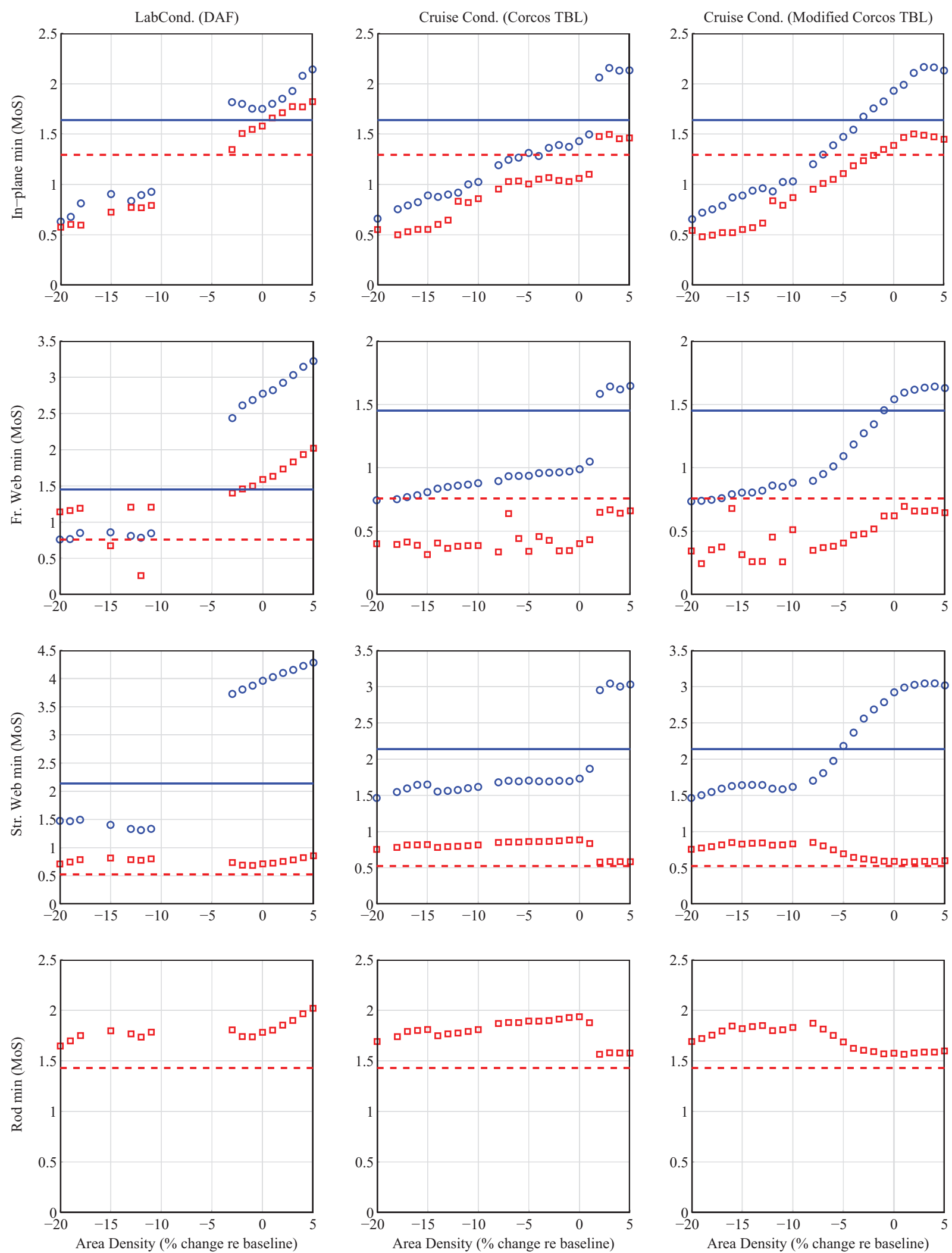

Fig. 11 Plots of minimum margins of safety relative to tension (o) and compression ( $\square$ ) allowables (Table 4) calculated with strain results from $2 P$ static pressurization analyses of Pareto optimal configurations. Baseline configuration results in tension (-) and compression (- -$)$ are also shown. Note that $y$ axis limits are varied.

The behavior of the frame webs in compression is of highest concern due to the relatively low MOS exhibited by the lower-weight Pareto configurations. Upon inspection of the models, some of these configurations exhibited local buckling at the tops of the frames near the center of the panel in the form of sinusoidal deformation with small lateral peak-peak deflections. This suggests that the design space may be too aggressive along the frame dimensions (frame spacing and height in particular) and may warrant additional investigation with a higher-fidelity $\mathrm{FE}$ model including threedimensional continuum elements representing the foam core and the addition of the pinch detail near the frame tops, both of which would tend to stabilize the frames under compression. 


\section{Conclusions}

An efficient approach to integrate vibroacoustic analysis at the early vehicle design stage has been proposed and exercised on a novel stitched and cocured composite structural concept known as PRSEUS. This approach is sufficiently flexible to account for different fluctuating pressure excitation fields and to evaluate the vibroacoustic impact of component level changes to PRSEUS configurations. Two major enabling aspects of the approach described here were the ability to quickly construct computational models given a set of panel configuration parameters and the use of response surrogate models to reduce the computational expense of resolving the multi-objective Pareto set with evolutionary algorithms. The results suggest that meaningful improvements can be made to the baseline PRSEUS configuration in terms of reduced weight, improved vibroacoustic performance, or both while maintaining positive margins of safety in the structure subject to the ultimate static pressurization load considered.

\section{Acknowledgments}

Support for this work was provided by the Environmentally Responsible Aircraft project of the Integrated Systems Research Program. The authors wish to thank Dawn Jegley and Richard Silcox (NASA Langley Research Center, Hampton, VA) and Alex Velicki (The Boeing Company, Huntington Beach, CA) for their helpful discussions during this work.

\section{References}

[1] Velicki, A., Thrash, P., and Jegley, D., "Airframe Development for the Hybrid Wing Body Aircraft," 47th AIAA Aerospace Sciences Meeting, AIAA Paper 2009-0932, Jan. 2009.

[2] Velicki, A., "Damage Arresting Composites for Shaped VehiclesPhase 1 Final Report," NASA CR-15932, 2009.

[3] Velicki, A., Yovanof, N., Baraja, J., Linton, K., Li, V., Hawley, A., Thrash, P., DeCoux, S., and Pickell, R., "Damage Arresting Composites for Shaped Vehicles—Phase 2 Final Report," NASA CR-216880, 2011.

[4] Linton, K. A., Velicki, A., Hoffman, K., Thrash, P., Pickell, R., and Turley, R., "PRSEUS Panel Fabrication Final Report," NASA CR2014-218149, 2014.

[5] Przekop, A., Wu, H. Y. T., and Shaw, P., "Nonlinear Finite Element Analysis of a Composite Non-Cylindrical Pressurized Aircraft Fuselage Structure," 55th AIAA/ASME/ASCE/AHS/ASC Structures, Structural Dynamics, and Materials Conference, AIAA Paper 2014-1064, Jan. 2014.

[6] "ABAQUS Version 6.12 Documentation, ABAQUS Analysis User's Manual,” Dassault Systèmes, Providence, RI, 2012.

[7] Lophaven, S. N., Nielsen, H. B., and Søndergaard, J., "DACE: A MATLAB Kriging Toolbox," Technical Univ. of Denmark, TR IMMTR-2002-12, Dept. of Informatics and Mathematical Modelling, Kongens Lyngby, 2002.

[8] Allen, A., and Przekop, A., "Vibroacoustic Characterization of a New Hybrid Wing-Body Fuselage Concept," Proceedings of the 2012 Internoise and ASME NCAD Meeting, American Soc. of Mechanical Engineers Paper IN12-321, Fairfield, NJ, Aug. 2012.

[9] Cunefare, K. A., Crane, S. P., Engelstad, S. P., and Powell, E. A., "ATool for Design Minimization of Aircraft Interior Noise," 2nd AIAA/CEAS Aeroacoustics Conference, AIAA Paper 1996-1702, May 1996.

[10] Fernholz, C. M., and Robinson, J. H., "The Influence of Lamination Angles on the Interior Noise Levels of an Aircraft," NASA TM-110250, Aug. 1996.
[11] Robinson, J. H., "Shape Optimization of Cylindrical Shell for Interior Noise," NASA TR-20040110716, 1999.

[12] Joshi, P., Mulani, S. B., Slemp, W. C. H., and Kapania, R. K., "VibroAcoustic Optimization of Turbulent Boundary Layer Excited Panel with Curvilinear Stiffeners," Journal of Aircraft, Vol. 49, No. 1, 2012, pp. 52-65. doi:10.2514/1.C031105

[13] Miller, T. S., Gallman, J. M., and Moeller, M. J., "Review of Turbulent Boundary Layer Models for Acoustic Analysis," 49th AIAA Aerospace Sciences Meeting, AIAA Paper 2011-1083, Jan. 2011.

[14] Mathur, G. P., Chin, C. L., and Simpson, M. A., "Transmission Loss of a Panel Excited by Turbulent Boundary Layer Pressure Field," Proceedings of Noise-Conference 4 96, Noise Control Foundation, New York, Sept. 1996, pp. 205-210.

[15] Orrenius, U., Cotoni, V., and Wareing, A., "Analysis of Sound Transmission Through Aircraft Fuselages Excited by Turbulent Boundary Layer or Diffuse Acoustic Pressure Fields," Proceedings of Inter-Noise 2009, Inst. of Noise Control Engineering Paper IN09-907, Reston, VA, Aug. 2009.

[16] Collery, O., Neple, P., and Guyader, J.-L., "Add-On Damping Patches Efficiency on Sound Transmission of a Thin Plate Under Aerodynamic or Acoustic Excitation," Proceedings of Inter-Noise 2009, Inst. of Noise Control Engineering Paper IN09-413, Reston, VA, Aug. 2009.

[17] Fahy, F., Sound and Structural Vibration: Radiation, Transmission, and Response, Academic Press, Oxford, 2007, pp. 159-168.

[18] Pierce, A. D., Acoustics: An Introduction to Its Physical Principles and Applications, Acoustical Soc. of America, Melville, NY, 1991, pp. 217-220.

[19] Cook, R. K., Waterhouse, R. V., Berendt, R. D., Edelman, S., and Thompson, M. C. Jr., "Measurement of Correlation Coefficients in Reverberant Sound Fields," Journal of the Acoustical Society of America, Vol. 27, No. 6, Nov. 1955, pp. 1072-1077. doi:10.1121/1.1908122

[20] Waterhouse, R. V., "Interference Patterns in Reverberant Sound Fields," Journal of the Acoustical Society of America, Vol. 27, No. 2, March 1955, pp. 247-258. doi:10.1121/1.1907509

[21] Corcos, G. M., "Resolution of Pressure in Turbulence," Journal of the Acoustical Society of America, Vol. 35, No. 2, Feb. 1963, pp. 192-199. doi:10.1121/1.1918431

[22] Hwang, Y. F., "A Discrete Model of Turbulence Loading Function for Computation of Flow-Induced Vibration and Noise," Proceedings of the ASME International Mechanical Engineering Congress and Exposition, American Soc. of Mechanical Engineers, Fairfield, NJ, Nov. 1998, pp. 389-395.

[23] Efimtsov, B. M., "Characteristics of the Field of Turbulent Wall Pressure Fluctuations at Large Reynolds Numbers," Akusticheskii Zhurnal, Vol. 28, July 1982, pp. 491-497.

[24] Schlichting, H., Boundary-Layer Theory, McGraw-Hill, New York, 1968 , pp. 566, 604, 617

[25] Velicki, A., Yovanof, N., Baraja, J., Mathur, G., Thrash, P., and Pickell, R., "PRSEUS Acoustic Panel Fabrication," NASA CR-217309, 2011.

[26] Lovejoy, A. E., Rouse, M., Linton, K. A., and Li, V., "Pressure Testing of a Minimum Gauge PRSEUS Panel," 52nd AIAA/ASME/ASCE/AHS/ ASC Structures, Structural Dynamics, and Materials Conference, AIAA Paper 2011-1813, April 2011.

[27] Sallaberry, C. J., Helton, J. C., and Hora, S. C., "Extension of Latin Hypercube Samples with Correlated Variables," Reliability Engineering and System Safety, Vol. 93, No. 7, 2008, pp. 1047-1059. doi:10.1016/j.ress.2007.04.005

[28] Oldenhuis, R., "GODLIKE - A Robust Single and Multi-Objective Optimizer," The MathWorks, Natick, MA, 2009, http://www.mathworks .com/matlabcentral/fileexchange/24838 [retrieved Sept. 2012]. 